\title{
An Algebraic Formulation for the Configuration Transformation of a Class of Reconfigurable Cube Mechanisms
}

\author{
Chin-Hsing Kuo, Jyun-Wei Su, and Lin-Chi Wu \\ Department of Mechanical Engineering, National Taiwan University of Science and Technology, \\ Taipei 106, Taiwan \\ Correspondence to: Chin-Hsing Kuo (chkuo717@mail.ntust.edu.tw)
}

Received: 6 September 2016 - Revised: 25 February 2017 - Accepted: 22 March 2017 - Published: 12 April 2017

\begin{abstract}
This paper presents an algebraic strategy for formulating the configuration transformation of a special class of reconfigurable cube mechanism (RCM) made by $2^{3}$ cyclically connected sub-cubes. The RCM studied here is kinematically equivalent to a spatial eight-bar linkage having eight transformable configurations. In this paper, the reconfiguration characteristics of the RCM are figured out first. Then, the initial configuration of the RCM is described by a joint-screw matrix, from which all the consecutive joint-screw matrices that represent the configuration transformation of the RCM can be derived. An illustrative example is provided to determine the eight joint-screw matrices of an RCM at an initial configuration. This reconfiguration formulation is further applied to enumerate all feasible topological configurations of such a special reconfigurable mechanism. The results show that, for such a special kind of reconfigurable cube mechanisms, there is only one feasible initial topological configuration for the RCM to perform a complete cycle of reconfiguration.
\end{abstract}

\section{Introduction}

There is a special class of reconfigurable cube mechanism (RCM) equivalent to an eight-bar closed-loop spatial linkage with cyclic reconfiguration, Fig. 1 . The mechanism can demonstrate eight different operation configurations, i.e., configuration $\mathrm{A}$ to $\mathrm{H}$ in Fig. 1, where nine different figures are exposed on the outer surface of the RCM. An earlier study (Kuo and Su, 2017) to this special mechanism has shown that the manipulation of this elegant artifact can be interpreted by using the mechanism theories in terms of variable mobility and isomorphism identification.

When investigating the topological properties of reconfigurable mechanisms, configuration transformation is one interesting topic to be explored. In literature, a couple of studies have made significant contributions to the configuration transformation analysis for some specific reconfigurable mechanisms. For example, Wohlhart (1996) introduced a special linkage, namely "kinematotropic linkage," that could permanently change its mobility by reconfigure the linkage into different working configurations. Dai and Rees
Jones (1999a, b, 2005) studied a special foldable/erectable mechanism and developed an EU-elementary matrix operation for formulating its topology transformation (Dai and Rees Jones, 2005). Zhang and Dai (2008, 2009) proposed an evolutionary reconfiguration algorithm of general spatial metamorphic mechanisms. Yan and Kang (2009a) studied the configuration transformation of variable topology mechanisms based on the concept of mapping function, leading to a general methodology for configuration synthesis of variable topology mechanisms (Yan and Kang, 2009b). Yan and Kuo $(2007,2009)$ put forward a systematic approach for configuration analysis and synthesis for general variable topology mechanisms where the topological reconfiguration can be described by graph (Yan and Kuo, 2006a), finite state machine (Yan and Kuo, 2006b), and screw matrix (Kuo and Yan, 2007). Ghrist and Peterson (2007) realized the reconfiguration of reconfigurable systems in robotics and biology by using state complex technique.

In addition to the general theories of reconfiguration, some tailor-made approaches for formulating the configuration transformation were available for several specific applica- 


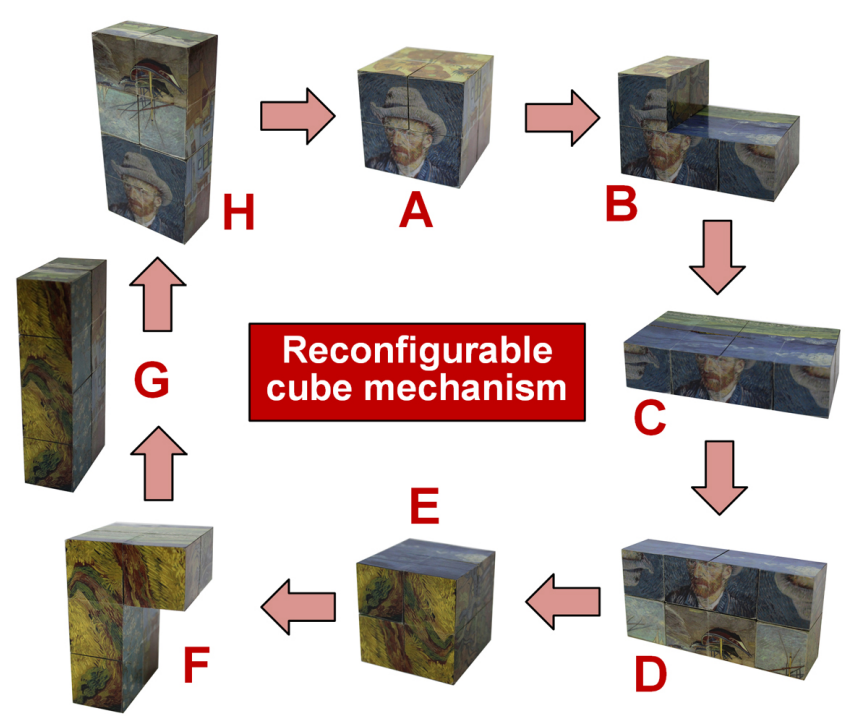

Figure 1. The reconfigurable cube mechanism (RCM).

tions. For instance, Liu and Dai (2002) investigated the folding process of packaging cartons, leading to a reconfiguration methodology and algorithm for the reconfigurable carton folding. Ding and Yang (2012) evaluated the geometry and reconfiguration principles of a special Mandala-type artifact and discussed its application for aerospace engineering. Wei et al. (2010, 2011) analyzed the configuration singularity and reconfiguration properties of a Hoberman switch-pitch ball. Ding et al. (2013) designed a deployable polyhedral linkage for which the changeable configuration was presented by joint screws. Ding and Lu (2013) analyzed the motion sequence and isomorphism of a chain-type cube mechanism. Gan et al. (2009, 2010, 2013a, b) proposed a metamorphic parallel mechanism whose configuration was changed via a special reconfigurable joint. Zhang et al. (2010a, 2012) presented other fantastic metamorphic parallel mechanisms via the concepts of variable-axis joints, origami folding (Zhang et al., 2010b), and kirigami (Zhang and Dai, 2014).

On the other hand, topology synthesis of reconfigurable mechanisms is also an interesting and challenging problem. In the past decades, topology synthesis of reconfigurable mechanisms has been attempted for several special mechanisms, e.g., the kinematotropic linkages (Galletti and Fanghella, 2001), metamorphic mechanisms (Zhang et al., 2008; Zhang and Dai, 2009), variable topology mechanisms (Yan and Kuo, 2009; Kuo and Chang, 2014; Shieh et al., 2011), and reconfigurable mechanisms (Kuo et al., 2009; Huang et al., 2010). However, those studies were mostly focused on the deployable or folding mechanisms. For the reconfigurable mechanisms formed by connected sub-cubes that we study here, its topology synthesis and enumeration tasks are still an open problem.

Therefore, the aim of this work is to develop an algebraic formulation for describing the configuration transformation of the RCM made by $2^{3}$ sub-cubes. Then, based on the developed formulation, all topologically non-isomorphic configurations of the RCMs are enumerated. In what follows, Sect. 2 firstly studies the reconfiguration characteristics of the RCM. Accordingly, Sect. 3 puts forward an algebraic computational procedure for representing the configuration transformation of the studied RCM. Based on this computational procedure, Sect. 4 briefs the enumeration of all possible topologically non-isomorphic configurations for the RCM with $2^{3}$ subcubes. Finally, Sect. 5 concludes the works and contribution of this paper.

\section{Configuration Characteristics}

By observing the RCM in Fig. 1, the topological and reconfiguration characteristics of the RCM can be concluded as follows.

\subsection{Topological Characteristics}

The topological characteristics of the RCM include:

1. The RCM is always a single-loop closed kinematic chain during reconfiguration.

2. All the sub-cubes are topologically similar class. In the $\mathrm{RCM}$, the links are all binary links, the joints are all revolute joints, and the linkage is a single-loop chain. Therefore, each link is topologically identical to each other, i.e., the links are similar class (Harary, 1964).

3. The orientation of each joint is not changed after the joint is reconfigured. Referring Fig. 4 in (Kuo and $\mathrm{Su}$, 2017), for example, when the blue sub-cube is grounded, the RCM can verify a series of configuration changes as shown from Fig. $4 a$ to $h$. Then, it can be verified that the orientation of each joint will remain the same in all configurations, even if the joint axis is displaced from some configuration to another one. For example, joint $c$ in the figure always points at the $z$ direction in the eight configurations.

4. Each configuration must have joints pointing at the $x$-, $y$-, and $z$-directions, respectively. Subject to point 3), since the RCM has $x$-, $y$-, and $z$-direction joints at any initial configuration, each configuration will possess joints corresponding to the three directions, respectively. This fact can be verified from Fig. 4 in (Kuo and $\mathrm{Su}, 2017)$.

5. Since all the joints are incident to the edges of the subcubes, all the joints form an orthogonal pattern in each configuration, i.e., they are either parallel or orthogonal to one another in each configuration.

6. In each configuration, there must have exactly two, two, and four joints pointing at the three axial directions, 
respectively. For example, there may have two joints pointing at the $z$-direction, two joints at the $x$-direction, and two joints at the $y$-direction in some configuration.

\subsection{Reconfiguration Characteristics}

The reconfiguration characteristics of the RCM include:

1. When any two joints are being coaxial, they will become a pair of workable joints, i.e., the degree of freedom of motion of the joint is not restrained by the configurations or the link shape.

2. The workable joints must appear in pairwise-there is no single workable joint on an axis.

3. When there exists a pair of workable joints in the configuration, this configuration is able to transform into the next one. For example, in Fig. 4h of (Kuo and Su, 2017), joints $(b, f)$ and $(d, h)$ are the only two pairs of workable joints. So joints $(b, f)$ or $(d, h)$ may be actuated to transfer configuration $H$ into $G$ or $A$, respectively.

4. The configuration of the RCM can be classified into "operation status" or "transition status." When the RCM is at an operation status, it has two or more pairs of workable joints that are pointing at two different orientations. On the other hand, when the RCM is at a transition status, it has only one pair of workable joints. Therefore, referring Fig. 4 in (Kuo and Su, 2017), configurations $\mathrm{A}, \mathrm{C}, \mathrm{D}, \mathrm{E}, \mathrm{G}$, and $\mathrm{H}$ are at operation status, whereas configurations $\mathrm{B}$ and $\mathrm{F}$ are at transition status.

5. The transformation from configuration $X$ to $Y(X$, $Y=\mathrm{A}, \mathrm{B}, \ldots, \mathrm{H})$ is called "forward" if it follows the transferring direction in the previous one. Otherwise, it is called a "backward" transformation. For example, Fig. 2 shows the reconfiguration sequence of the RCM of Fig. 4 in (Kuo and Su, 2017). The circles denote the configurations $\mathrm{A}$ to $\mathrm{H}$ and two trivial configurations $\mathrm{B}^{\prime}$ and $\mathrm{F}^{\prime}$ (that are equivalent to configurations $\mathrm{B}$ and $\mathrm{F}$, respectively). Notation $(\alpha, \beta)$ between two circles indicates the working joints under which the configurations can be transformed. For example, configuration $\mathrm{A}$ can be transformed to $\mathrm{B}$ by operating joints $(a, g)$ and vice versa. Therefore, as shown on the figure, configuration $A$ has three pairs of workable joints, i.e., $(a, g),(d, h)$, and $(c, e)$, and it can be transformed into different configurations as different workable joints are operated. So, if the RCM is reconfigured via a sequence of $\mathrm{A} \rightarrow \mathrm{B} \rightarrow \mathrm{C} \rightarrow \mathrm{D}$, the transformations from $\mathrm{B}$ to $\mathrm{C}$ and from $\mathrm{C}$ to $\mathrm{D}$ are both forward. If the $\mathrm{RCM}$ is reconfigured, for example, via a sequence of $\mathrm{A} \rightarrow \mathrm{B} \rightarrow \mathrm{C} \rightarrow \mathrm{D} \rightarrow \mathrm{C}$, the transformation from $\mathrm{D}$ to $\mathrm{C}$ is backward. As shown in the figure, it is noticed that configuration $\mathrm{A}$ can be transformed to $\mathrm{C}$ via sequence $\mathrm{A} \rightarrow \mathrm{B} \rightarrow \mathrm{C}$ or $\mathrm{A} \rightarrow \mathrm{B}^{\prime} \rightarrow \mathrm{C}$. For sequence $\mathrm{A} \rightarrow \mathrm{B} \rightarrow \mathrm{C}$ the working joints are $(a, g)$ and $(c, e)$ in turn, whereas for sequence $\mathrm{A} \rightarrow \mathrm{B}^{\prime} \rightarrow \mathrm{C}$ the working joints are $(c, e)$ and $(a, g)$ inturn. Obviously, the sets of the working joints for these two sequences are identical but have different orders of actuation. It is further observed that the two transition configurations between $\mathrm{A}$ and $\mathrm{C}$, i.e. $\mathrm{B}$ and $\mathrm{B}^{\prime}$, are configurationally isomorphic (Kuo and $\mathrm{Su}$, 2017) in essential. Therefore, configuration $B^{\prime}$ is trivial as it plays the same role of configuration $\mathrm{B}$. This situation also happens between configurations $\mathrm{E}$ and $\mathrm{G}$ where the trivial configuration $\mathrm{F}^{\prime}$ plays the same function of configuration $\mathrm{F}$.

6. The operation of a working joint is either a forward or a backward operation. A configuration is said under a forward operation when its working joints are different from the ones in the previous two transformations ${ }^{1}$. On the other hand, a configuration is said under a backward operation when its working joints are as same as the ones in any of the previous two configurations. For example, if the RCM is reconfigured at a sequence of $\mathrm{A} \rightarrow \mathrm{B} \rightarrow \mathrm{C} \rightarrow \mathrm{D}$, then the operation of joints $(d, h)$ between $\mathrm{C}$ and $\mathrm{D}$ is a forward operation since it is different from the two previous operations $(c, e)$ and $(a, g)$ in this reconfiguring sequence. On the other hand, if the RCM is reconfigured by $\mathrm{B} \rightarrow \mathrm{A} \rightarrow \mathrm{B}^{\prime} \rightarrow \mathrm{C}$, then the operation of joints $(a, g)$ between $\mathrm{B}^{\prime}$ and $\mathrm{C}$ is a backward operation since it is identical to the second previous operation between configurations $\mathrm{B}$ and $\mathrm{A}$.

7. A backward operation of the working joints will induce a repeated transformation or reverse the direction of transformation of the reconfiguration. For instance, in the previous example, the operation of joints $(a, g)$ between $\mathrm{B}^{\prime}$ and $\mathrm{C}$ is a backward operation for transformation $\mathrm{B} \rightarrow \mathrm{A} \rightarrow \mathrm{B}^{\prime} \rightarrow \mathrm{C}$. This operation leads the RCM into configuration $C$, where the RCM has three options of the workable joints: $(c, e),(d, h)$, and $(a, g)$. If joints $(c, e)$ are selected as the working joints for configuration $\mathrm{C}$, the configuration will be changed from $\mathrm{C}$ to $\mathrm{B}$, where either the transformation $\mathrm{B} \rightarrow \mathrm{A}$ will be repeated or the reverse transformation $\mathrm{C} \rightarrow \mathrm{B}$ will happen. Alternatively, if either joint $(d, h)$ or $(a, g)$ are selected as the working joints for configuration $\mathrm{C}$, then a reverse transformation (i.e., the transformation in the main loop will be reversed from counterclockwise to clockwise or the transformation will be reversed from $\mathrm{B}^{\prime} \rightarrow \mathrm{C}$ to $\mathrm{C} \rightarrow \mathrm{B}^{\prime}$ ) will be resulted.

8. Each configuration has only one forward operation for forming a non-repeated, cyclic transformation. This property can be verified from Fig. 2 .

\footnotetext{
${ }^{1}$ The two coaxial working joints of a configuration can point at either $x$-, $y$-, or $z$-directions only. So, the working joints merely need to compare with those in the previous two transformations.
} 


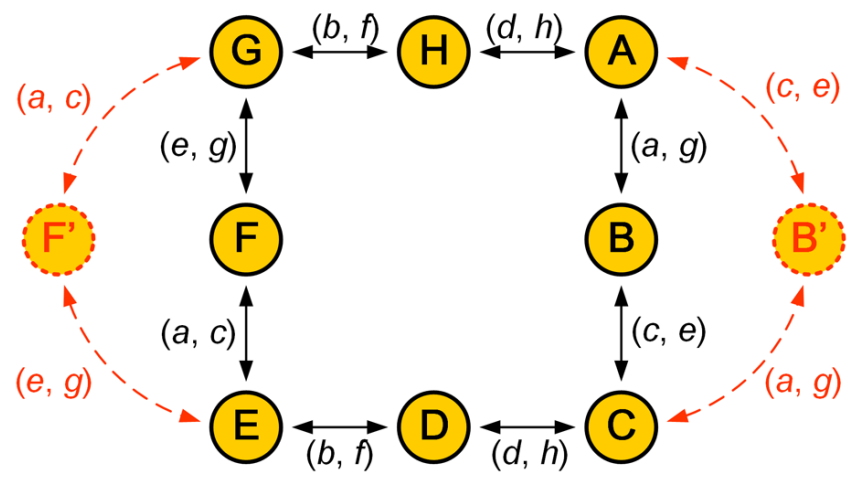

Figure 2. The reconfiguration sequence of the RCM.

\section{Configuration Transformation}

The configuration transformation of the RCM is an interesting problem In our previous study (Kuo and Su, 2017), we have shown that the configurations of the RCM can be represented by a matrix of joint screws. In what follows, a computational procedure is presented for deriving all the configurations of the RCM based on a given screw matrix of the initial configuration.

\subsection{A Computational Procedure for Formulating the Configuration Transformation Process}

The flowchart of the computational procedure for formulating the configuration transformation process is given in Fig. 3. The detailed procedure is introduced as follows.

\subsubsection{Defining the referencing coordinate system}

When an initial configuration of the RCM is given, a Cartesian coordinate system is attached to some link as a referencing coordinate system for the reconfiguration. For convenience, the origin of the system is set to some corner of the sub-cube, and the three coordinate axes are to point along the edges of the cube. For example, Fig. $4 \mathrm{a}$ is a given initial configuration of an RCM (same as Fig. 5a in Kuo and Su, 2017) and a coordinate system is attached onto link 2 as shown. Note that the arrangement of the coordinate system is independent of the derived results, i.e., it can be arbitrarily set to any links and any corner.

\subsubsection{Determining the initial joint-screw matrix}

Now, the joint-screw matrix $\Pi_{\text {initial }}$ (Kuo and Su, 2017) can be written with respect to the defined coordinate system at the initial configuration. For example, the joint-screw matrix

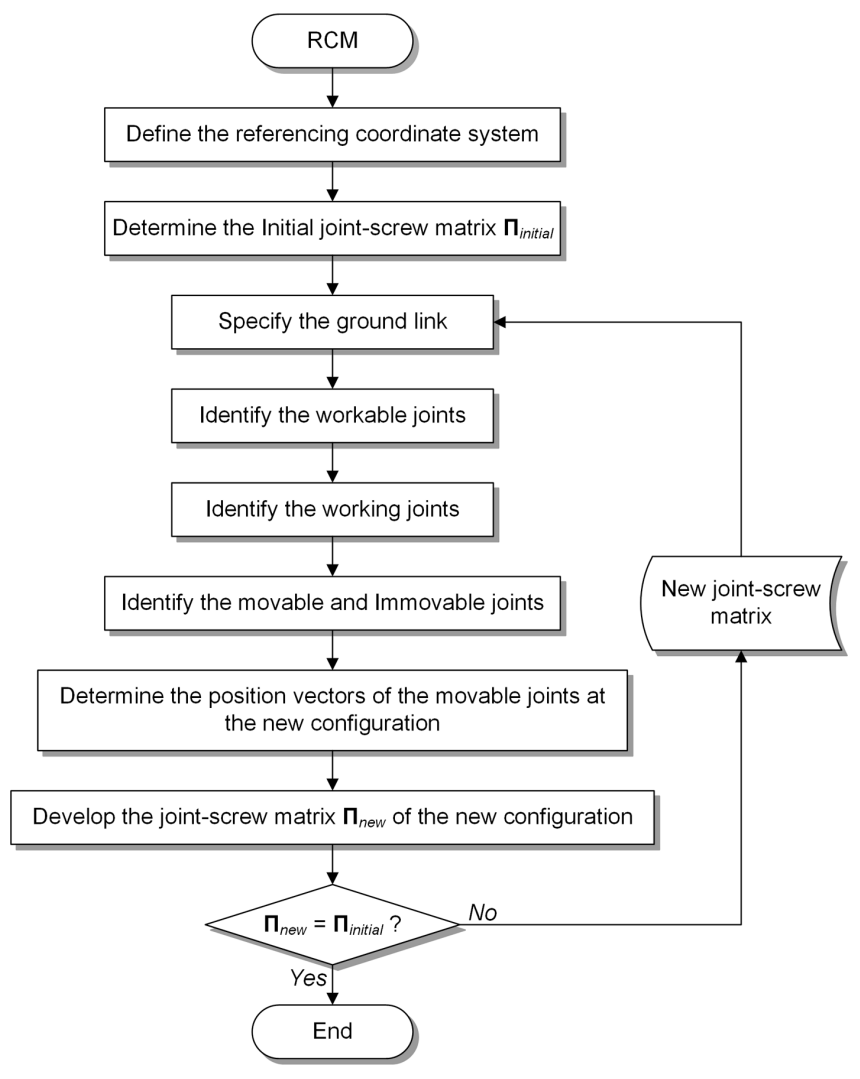

Figure 3. A computational procedure for determining all feasible configurations of the RCM.
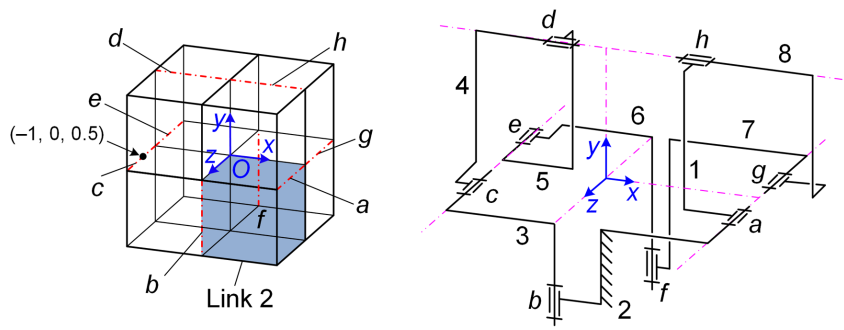

Figure 4. The RCM at the initial configuration. (a) The RCM; (b) equivalent linkage.

for the configuration in Fig. 4a is:

$\Pi_{\text {initial }}=\left[\begin{array}{l}\boldsymbol{S}_{a}^{T} \\ \boldsymbol{S}_{b}^{T} \\ \boldsymbol{S}_{c}^{T} \\ \boldsymbol{S}_{d}^{T} \\ \boldsymbol{S}_{e}^{T} \\ \boldsymbol{S}_{f}^{T} \\ \boldsymbol{S}_{g}^{T} \\ \boldsymbol{S}_{h}^{T}\end{array}\right]=\left[\begin{array}{llllll}0 & 0 & 1 & 0 & -1 & 0 \\ 0 & 1 & 0 & -1 & 0 & 0 \\ 0 & 0 & 1 & 0 & 1 & 0 \\ 1 & 0 & 0 & 0 & 0 & -1 \\ 0 & 0 & 1 & 0 & 1 & 0 \\ 0 & 1 & 0 & 1 & 0 & 0 \\ 0 & 0 & 1 & 0 & -1 & 0 \\ 1 & 0 & 0 & 0 & 0 & -1\end{array}\right]$

Where $S_{a}^{T}$ represents the transpose of the screw vector of joint $a$ and so does to other joints. Note that, as explained in 


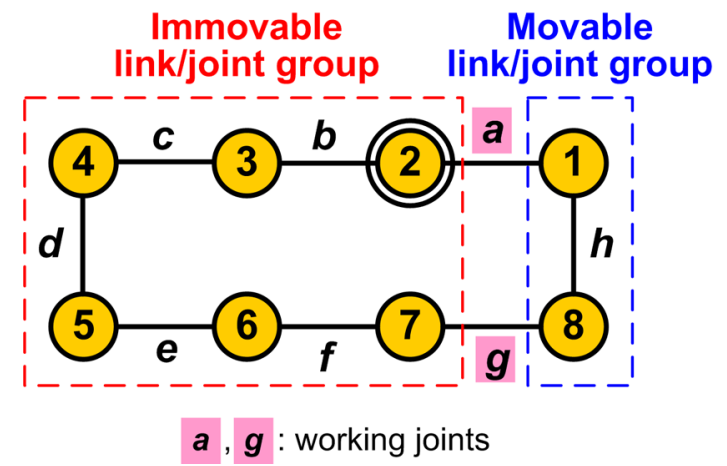

Figure 5. The movable and immovable link/joint groups.

Kuo and Su (2017), each sub-cube is defined as a unit cube and all the unit vectors $\mathbf{s}$ for the joint screws should point at the positive $x, y$, and $z$ directions, respectively.

\subsubsection{Specifying the ground link}

Since the RCM linkage has no specific ground link, one link should be grounded to be the reference of the relative motions of the other sub-cubes. Any link of the RCM can be selected as the ground. For example, for the RCM in Fig. 4a, link 2 is selected as the ground link. Accordingly, the RCM becomes a linkage mechanism as depicted in Fig. $4 \mathrm{~b}$.

\subsubsection{Identifying the workable joints}

As stated in Sect. 2, a workable joint is a joint whose degree of freedom of motion is not restrained by the configuration or the link shapes. According to the reconfiguration characteristics described in Sect. 2, when two joints become coaxial, they will together form a pair of workable joints. So our next step is to identify the group of the workable joints from the joint-screw matrix. For example, it can be easily identified from Eq. (1) that vectors $\left(\boldsymbol{S}_{a}, \boldsymbol{S}_{g}\right),\left(\boldsymbol{S}_{c}, \boldsymbol{S}_{e}\right)$, and $\left(\boldsymbol{S}_{d}, \boldsymbol{S}_{h}\right)$ form three groups with identical vectors within it. Therefore, it concludes that there are three groups of workable joints, $(a, g),(c, e)$, and $(d, h)$, at the shown configuration.

\subsubsection{Identifying the working joints}

Now one group of the workable joints is chosen as the group of working joints for actuating the RCM at the configuration. In order to derive all follow-up feasible configurations of the $\mathrm{RCM}$, i.e., a cyclic reconfiguration without repeated configurations, only the group of working joints that is at forward operation as introduced in Sect. 2 can be chosen. To do this, the selected working joint group should be compared with the working joint groups in the previous two configurations. If the selected working joint group is not as same as that in the previous two configurations, it will be a feasible working joint group. This comparison should be continued until the working joint group has been identified. Since each configuration has only one forward operation, the selection result of the working joint group will be unique. If there are no previous configurations to be compared, e.g., the current configuration is the first or second configuration, this check can be ignored. For example, for the configuration in Fig. 4, joint group $(a, g)$ is selected as the working joints.

\subsubsection{Identifying the movable and immovable joints}

After the working joint group is identified, all the remaining joints can be divided into two kinds, the movable joints and immovable joints. A movable joint means that its joint axis will be displaced as the working joints are actuated. Reversely, the location of the joint axis of an immovable joint will be unchanged when the working joints are functioning. The identification of movable and immovable joints is illustrated via the topological graph in Fig. 5. In this graph, the vertices represent the links with their numbering and the edges are the joints with their labeling. The ground link, link 2 , is labeled with a concentric circle. In the previous step, joint group $(a, g)$ was identified as the working joints, so the cyclic connection of the vertices and edges in the graph can be divided by joint $(a, g)$ so as to form two groups, i.e., the two dashed-line blocks. Then, the group that contains the ground link is the immovable joint/link group and the other one is the movable joint/link group. So, in this configuration, the movable joints are joint $h$ only, and the immovable joints are joints $a, b, c, d, e, f$, and $g$. The above identification of the movable joints can be manipulated by the joint-screw matrix by using the following algorithm:

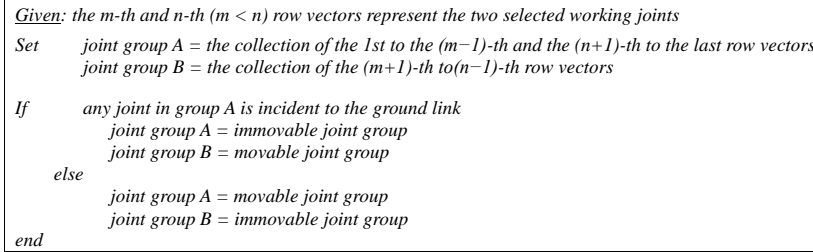

3.1.7 Deriving the position vectors of the movable joints at the new configuration

When a pair of joints $\left(J_{w 1}, J_{w 2}\right)$ is selected as the working joints at certain configuration, a couple of joints $\left(J_{m 1}, J_{m 2}\right.$, $\left.\ldots, J_{m i}\right)$ will become the movable joints of a forward transformation for the configuration. The next step is to determine the new screw vectors of these movable joints in the next configuration. Since reconfiguring a joint will not change its orientation (as analyzed in Sect. 2), only the position vector of the joint screw will be changed after reconfiguration. In what follows, the derivation of the new position vectors of the movable joints at the next configuration will be illustrated. 
Table 1. Orientation and position vectors of the RCM at the new configuration.

\begin{tabular}{lrr}
\hline Joint & $\begin{array}{r}\text { Orientation } \\
\text { vector }\end{array}$ & $\begin{array}{r}\text { Position } \\
\text { vector }\end{array}$ \\
\hline$a$ & {$[0,0,1]^{T}$} & {$[1,0,0.5]^{T}$} \\
$b$ & {$[0,1,0]^{T}$} & {$[0,-0.5,1]^{T}$} \\
$c$ & {$[0,0,1]^{T}$} & {$[-1,0,0.5]^{T}$} \\
$d$ & {$[1,0,0]^{T}$} & {$[-0.5,1,0]^{T}$} \\
$e$ & {$[0,0,1]^{T}$} & {$[-1,0,-0.5]^{T}$} \\
$f$ & {$[0,1,0]^{T}$} & {$[0,-0.5,-1]^{T}$} \\
$g$ & {$[0,0,1]^{T}$} & {$[1,0,-0.5]^{T}$} \\
$h$ & {$[1,0,0]^{T}$} & {$[1.5,-1,0]^{T}$} \\
\hline
\end{tabular}

The position vector of a joint in the unit RCM is measured from the origin of the reference system to the middle point of the joint. For example, the position vector of joint $c$ in Fig. 4 is $[-1,0,0.5]^{T}$. Assume that a working joint $J_{w}$ has a position vector $\left[p_{x}, p_{y}, p_{z}\right]^{T}$ and some movable joint $J_{m}$ has a position vector $\left[q_{x}, q_{y}, q_{z}\right]^{T}$ at the current configuration. If a working joint $J_{w}$ points at the $\alpha$-direction $(\alpha=x, y$, or $z$ ), the new position vector, $\left[\bar{q}_{x}, \bar{q}_{y}, \bar{q}_{z}\right]^{T}$, of the movable joint $J_{m}$ at the next configuration can be calculated by

$\bar{q}_{\alpha}=q_{\alpha}$

$\bar{q}_{\beta}=2 p_{\beta}-q_{\beta}$

where $\beta$ represents the two axial directions other than $\alpha$. For example, it is known that joint $a$ is the working joint and joint $h$ the movable joint at the configuration in Fig. 5. So the position vector for joint $a$ can be written as $\left[p_{x}^{a}, p_{y}^{a}\right.$, $\left.p_{z}^{a}\right]^{T}=[1,0,0.5]^{T}$, and the position vector for joint $h$ as $\left[q_{x}^{h}\right.$, $\left.q_{y}^{h}, q_{z}^{h}\right]^{T}=[0.5,1,0]^{T}$. Since the working joint points at the $z$-direction $(\alpha=z)$, the new position vector for joint $h$ after reconfiguration can derived by Eq. (2) as

$$
\left[\begin{array}{l}
\bar{q}_{x}^{h} \\
\bar{q}_{y}^{h} \\
\bar{q}_{z}^{h}
\end{array}\right]=\left[\begin{array}{l}
2 p_{x}^{a}-q_{x}^{h} \\
2 p_{y}^{a}-q_{y}^{h} \\
q_{z}^{a}
\end{array}\right]=\left[\begin{array}{l}
2 \times 1-0.5 \\
2 \times 0-1 \\
0
\end{array}\right]=\left[\begin{array}{l}
1.5 \\
-1 \\
0
\end{array}\right]
$$

By following this logic, the new position vectors of all the joints can be derived as summarized in Table 1 .

\subsubsection{Developing the joint-screw matrix of the new configuration}

After obtaining the new position vectors of all movable joints, the joint-screw matrix of the new configuration can be constructed. First, the joint screws of the immovable joints will be the same in the new configuration. Second, for the moveable joints, the joint orientations will be invariant in all configurations and the position vectors of the joints have been derived in the previous step. So the joint-screw matrix $\Pi_{\text {new }}$ can be written accordingly. For example, the joint-
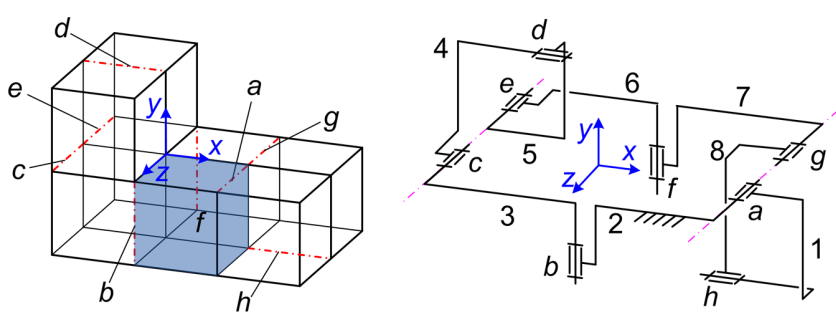

Figure 6. The RCM at the new configuration. (a) The RCM; (b) equivalent linkage (Kuo and $\mathrm{Su}, 2017$ ).
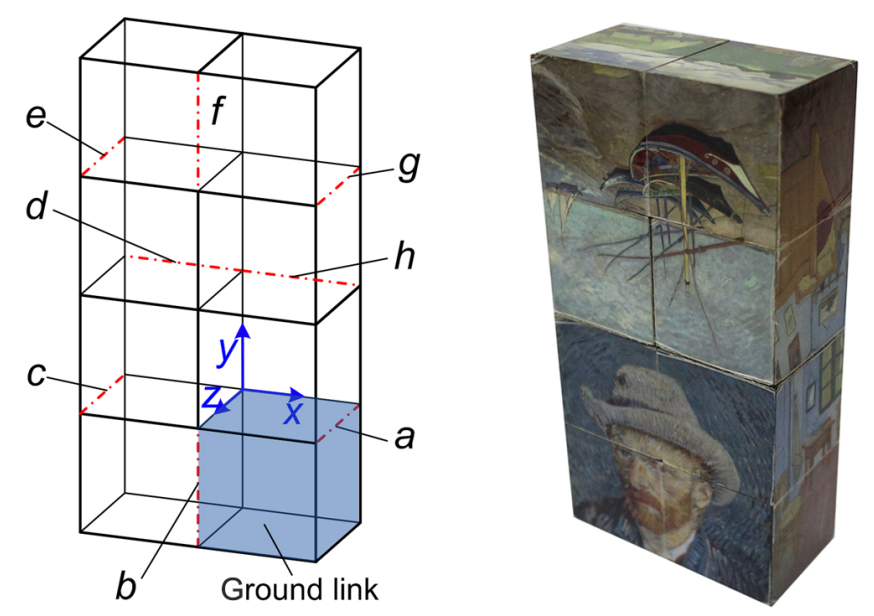

Figure 7. Initial configuration of the illustrative RCM. (a) Mechanism drawing (Kuo and Su, 2017); (b) physical model.

screw matrix of the new configuration in Fig. 4 is

$\Pi_{\text {new }}=\left[\begin{array}{l}\boldsymbol{S}_{a}^{T} \\ \boldsymbol{S}_{b}^{T} \\ \boldsymbol{S}_{c}^{T} \\ \boldsymbol{S}_{d}^{T} \\ \boldsymbol{S}_{e}^{T} \\ \boldsymbol{S}_{f}^{T} \\ \boldsymbol{S}_{g}^{T} \\ \boldsymbol{S}_{h}^{T}\end{array}\right]=\left[\begin{array}{llllll}0 & 0 & 1 & 0 & -1 & 0 \\ 0 & 1 & 0 & -1 & 0 & 0 \\ 0 & 0 & 1 & 0 & 1 & 0 \\ 1 & 0 & 0 & 0 & 0 & -1 \\ 0 & 0 & 1 & 0 & 1 & 0 \\ 0 & 1 & 0 & 1 & 0 & 0 \\ 0 & 0 & 1 & 0 & -1 & 0 \\ 1 & 0 & 0 & 0 & 0 & 1\end{array}\right]$

It is verified that this joint-screw matrix corresponds to the next configuration of the RCM, Fig. 6.

\subsubsection{Distinguishing the derived matrix with the one for the initial configuration}

After the joint-screw matrix $\Pi_{\text {new }}$ of the new configuration is determined, it should be compared with the joint-screw matrix, $\Pi_{\text {initial }}$, of the initial configuration. If they are not identical, then this matrix can be used to compute the screw matrix for its next configuration and so on. Oppositely, if the resulted matrix and the one from the initial configuration are identical, it means that the computation has verified all joint- 


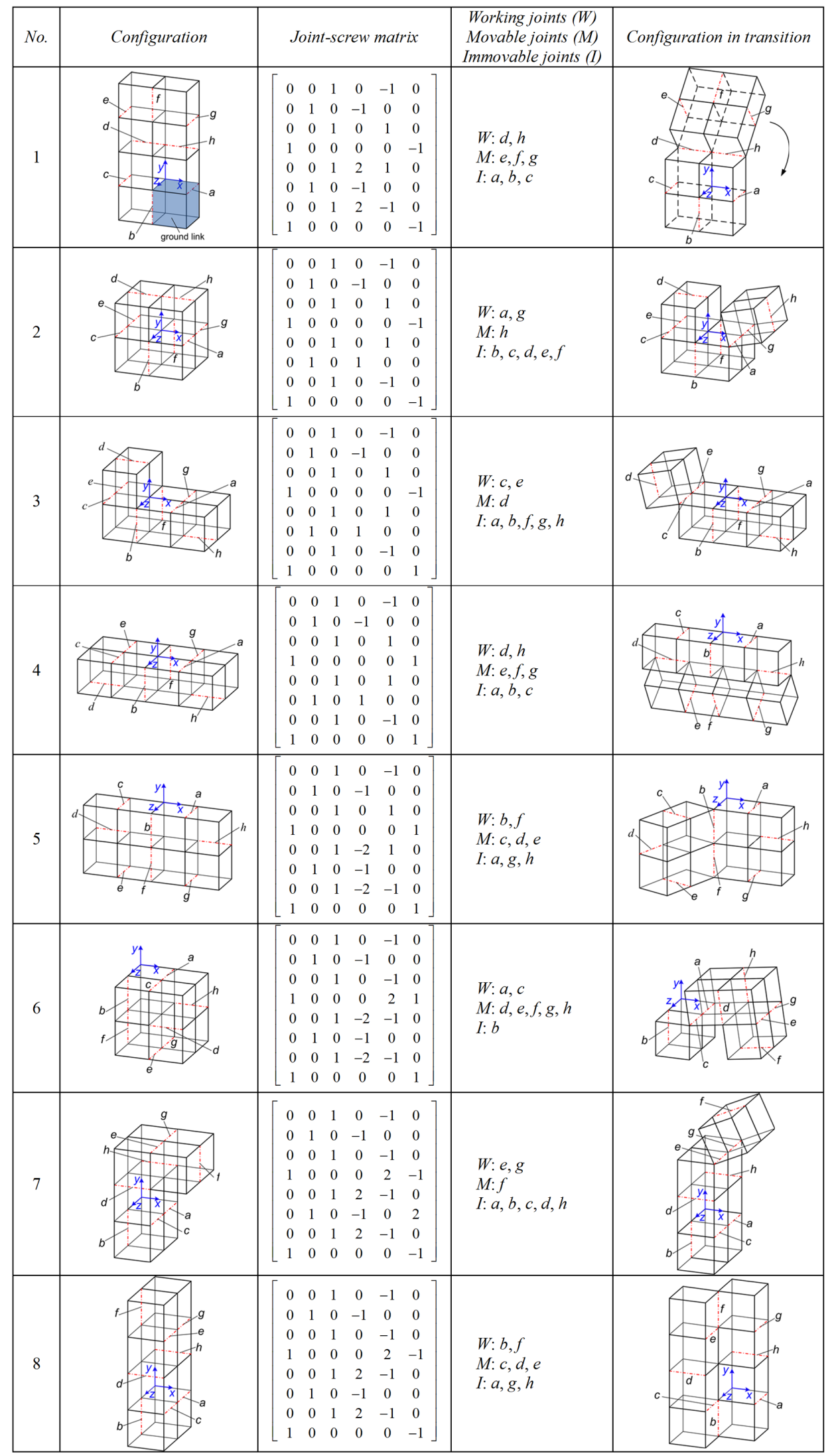

Figure 8. Computation results for the configuration transformation of the illustrative RCM. 
screw matrices for a cycle of the reconfiguration. In this case, the computation procedure can be terminated.

\subsection{Example}

Here the presented computational procedure is illustrated by taking Fig. 7 as an example. Figure 7 shows the initial configuration of an RCM to be manipulated. For illustration, the lower-right sub-cube is selected as the ground link in each following configuration. The computation results are summarized in Fig. 8.

\section{Topological Enumeration}

Based on the configuration transformation algorithm proposed above, all possible topological configurations of the RCMs with $2^{3}$ sub-cubes can be enumerated. The synthesized RCMs should have the same cubic geometry as the existing design and should be able to complete cyclic configuration transformation. A computational enumeration procedure has been introduced in our another work ( $\mathrm{Wu}$ and Kuo, 2015). The major steps for the enumeration is summarized as follows. First, all possible connecting sequences among the eight sub-cubes with rotational joints are enumerated. Next, according to some observed joint arrangement rules, the synthesized connecting sequences are examined to see whether or not it can represent a feasible mechanism. Then, the configurational isomorphism of the resulted configurations is further examined. Last, by applying the configuration transformation algorithm proposed in this paper, the cyclic reconfiguration ability for the RCMs is verified. It turns out that only one design can perform a complete cycle of configuration transformation. For more detailed enumeration procedure, one can refer to (Wu and Kuo, 2015).

\section{Conclusions}

A computational procedure was presented for formulating the configuration transformation of the RCM with $2^{3}$ subcubes. The reconfiguration characteristics of the RCM were first investigated. According to these characteristics, the configuration transformation of the RCM can be described by the manipulation on its joint-screw matrix. That is, given by one initial configuration, the joint-screw matrices of all its following transformed configurations in the RCM can be derived. The result further leads to the enumeration of all possible topological configurations of such an RCM. The result showed that when the RCM is made by $2^{3}$ sub-cubes, there is only one feasible initial topological configuration for the RCM to perform a complete cycle of reconfiguration, which is exactly the existing design.

Competing interests. The authors declare that they have no conflict of interest.
Edited by: X. Ding

Reviewed by: three anonymous referees

\section{References}

Dai, J. S. and Rees Jones, J.: Configuration Transformations in Metamorphic Mechanisms of Foldable/Erectable Kinds, Proceedings of the 10th World Congress on the Theory of Machines and Mechanisms, Oulu, Finland, 20-24 June, 1999a.

Dai, J. S. and Rees Jones, J.: Mobility in Metamorphic Mechanisms of Foldable/Erectable Kinds, ASME Journal of Mechanical Design, 121, 375-382, 1999b.

Dai, J. S. and Rees Jones, J.: Matrix Representation of Topological Changes in Metamorphic Mechanisms, ASME Journal of Mechanical Design, 127, 837-840, 2005.

Ding, X. and Lu, S.: Fundamental Reconfiguration Theory of Chain-Type Modular Reconfigurable Mechanisms, Mech. Mach. Theory, 70, 487-507, 2013.

Ding, X. and Yang, Y.: Reconfiguration Theory of Mechanism From a Traditional Artifact, ASME Journal of Mechanical Design, 132, 114501, doi:10.1115/1.4002692, 2012.

Ding, X., Yang, Y., and Dai, J. S.: Design and Kinematic Analysis of a Novel Prism Deployable Mechanism, Mech. Mach. Theory, 63, 35-49, 2013.

Galletti, C. and Fanghella, P.: Single-Loop Kinematotropic Mechanisms, Mech. Mach. Theory, 36, 743-761, 2001.

Gan, D., Dai, J. S., and Liao, Q.: Mobility Change in Two Types of Metamorphic Parallel Mechanisms, ASME Journal of Mechanisms and Robotics, 1, 041007, doi:10.1115/1.3211023, 2009.

Gan, D., Dai, J. S., and Liao, Q.: Constraint Analysis on Mobility Change of a Novel Metamorphic Parallel Mechanism, Mech. Mach. Theory, 45, 1864-1876, 2010.

Gan, D., Dai, J. S., Dias, J., and Seneviratne, L.: Unified Kinematics and Singularity Analysis of a Metamorphic Parallel Mechanism With Bifurcated Motion, ASME Journal of Mechanisms and Robotics, 5, 031004, doi:10.1115/1.4024292, 2013a.

Gan, D., Dai, J. S., Dias, J., and Seneviratne, L.: Reconfigurability and Unified Kinematics Modeling of a 3rTPS Metamorphic Parallel Mechanism with Perpendicular Constraint Screws, Robot. Cim.-Int. Manuf., 29, 121-128, 2013b.

Ghrist, R. and Peterson, V.: The Geometry and Topology of Reconfiguration, Adv. Appl. Math., 38, 302-323, 2007.

Harary, F.: Combinatorial Problems in Graphical Enumeration, in: Applied Combinatorial Mathematics, edited by: Beckenbach, E. F., J. Wiley, New York, NY, 1964.

Huang, H., Li, B., Liu, R., and Deng, Z.: Type Synthesis of Deployable/Foldable Articulated Mechanisms, 2010 International Conference on Mechatronics and Automation (ICMA), Xi' an, China, 4-7 August, 2010.

Kuo, C.-H. and Yan, H.-S.: On the Mobility and Configuration Singularity in Mechanisms with Variable Topologies, ASME Journal of Mechanical Design, 129, 617-624, 2007.

Kuo, C.-H. and Chang, L.-Y.: Structure Decomposition and Homomorphism Identification of Planar Variable Topology Mechanisms, ASME Journal of Mechanisms and Robotics, 6, 021002, doi:10.1115/1.4026336, 2014. 
Kuo, C.-H. and Su, J.-W.: Configuration Analysis of a Reconfigurable Cube Mechanism: Mobility and Configurational Isomorphism, Mech. Mach. Theory, 107, 369-383, 2017.

Kuo, C.-H., Dai, J. S., and Yan, H.-S.: Reconfiguration Principles and Strategies for Reconfigurable Mechanisms, ASME/IFToMM International Conference on Reconfigurable Mechanisms and Robots (ReMAR 2009), London, United Kingdom, 2009,

Liu, H. and Dai, J. S.: Carton Manipulation Analysis Using Configuration Transformation, Proceedings of the Institution of Mechanical Engineers, Part C, J. Mech. Eng. Sci., 216, 543-555, 2002

Shieh, W.-B., Sun, F., and Chen, D.-Z.: On the Operation Space and Motion Compatibility of Variable Topology Mechanisms, ASME Journal of Mechanisms and Robotics, 3, 021007, doi:10.1115/1.4003579, 2011.

Wei, G., Ding, X., and Dai, J. S.: Mobility and Geometric Analysis of the Hoberman Switch-Pitch Ball and Its Variant, ASME Journal of Mechanisms and Robotics, 2, 031010, doi:10.1115/1.4001730, 2010.

Wei, G., Ding, X., and Dai, J. S.: Geometric Constraint of an Evolved Deployable Ball Mechanism, Journal of Advanced Mechanical Design, Systems and Manufacturing, 5, 302-314, 2011.

Wohlhart, K.: Kinematotropic Linkages, in: Recent Advances in Robot Kinematics, edited by: Lenarčič, J. and Parenti-Castelli, V., Kluwer Academic Publishers, Dordrecht, Netherlands, 359368, 1996.

Wu, L.-C. and Kuo, C.-H.: Enumerating the Topological Configurations of the Reconfigurable Cube Mechanism with Eight Subcubes, The 3rd ASME/IFToMM International Conference on Reconfigurable Mechanisms and Robots (ReMAR 2015), Beijing, China, 20-22 July, 2015.

Yan, H.-S. and Kuo, C.-H.: Topological Representations and Characteristics of Variable Kinematic Joints, ASME Journal of Mechanical Design, 128, 384-391, 2006a.

Yan, H.-S. and Kuo, C.-H.: Representations and Identifications of Structural and Motion State Characteristics of Mechanisms with Variable Topologies, T. Can. Soc. Mech. Eng., 30, 19-40, 2006b.
Yan, H.-S. and Kang, C.-H.: Configuration Transformations of Mechanisms with Variable Topologies, J. Chin. Soc. Mech. Eng., 30, 311-321, 2009a.

Yan, H.-S. and Kang, C.-H.: Configuration Synthesis of Mechanisms with Variable Topologies, Mech. Mach. Theory, 44, 896911, 2009b.

Yan, H.-S. and Kuo, C.-H.: Structural Analysis and Configuration Synthesis of Mechanisms with Variable Topologies, ASME/IFToMM International Conference on Reconfigurable Mechanisms and Robots, London, United Kingdom, 2009,

Zhang, K. and Dai, J. S.: A Kirigami-Inspired 8R Linkage and Its Evolved Overconstrained 6R Linkages With the Rotational Symmetry of Order Two, ASME Journal of Mechanisms and Robotics, 6, 021007, doi:10.1115/1.4026337, 2014.

Zhang, K., Dai, J. S., and Fang, Y.: Topology and Constraint Analysis of Phase Change in the Metamorphic Chain and Its Evolved Mechanism, ASME Journal of Mechanical Design, 132, 121001, doi:10.1115/1.4002691, 2010a.

Zhang, K., Fang, Y., Fang, H., and Dai, J. S.: Geometry and Constraint Analysis of the Three-Spherical Kinematic Chain Based Parallel Mechanism, ASME Journal of Mechanisms and Robotics, 2, 031014, doi:10.1115/1.4001783, 2010b.

Zhang, K., Dai, J. S., and Fang, Y.: Geometric Constraint and Mobility Variation of Two $3 \mathrm{~S}_{v} \mathrm{PS}_{v}$ Metamorphic Parallel Mechanisms, ASME Journal of Mechanical Design, 135, 011001, doi:10.1115/1.4007920, 2012.

Zhang, L. and Dai, J. S.: Genome Reconfiguration of Metamorphic Manipulators Based on Lie Group Theory, ASME 2008 International Design Engineering Technical Conferences (IDETC 2008), Brooklyn, New York, USA, 2008.

Zhang, L. and Dai, J. S.: Reconfiguration of Spatial Metamorphic Mechanisms, ASME Journal of Mechanisms and Robotics, 1, 011012, doi:10.1115/1.2963025, 2009.

Zhang, L., Wang, D., and Dai, J. S.: Biological Modeling and Evolution Based Synthesis of Metamorphic Mechanisms, ASME Journal of Mechanical Design, 130, 072303, doi:10.1115/1.2900719, 2008. 